\title{
Habitat marking: Parallel genetic divergence in two Drosophila species
}

\author{
P. A. Parsons and A. A. Hoffmann*
}

Department of Genetics and Human Variation, La Trobe University, Bundoora, 3083, Victoria, Australia.

\begin{abstract}
Male populations of both the sibling species $D$. melanogaster and $D$. simulans are preferentially attracted to sites marked by the residual odours of females derived from their own geographic populations (Melbourne vs. Townsville), when cultured under uniform laboratory conditions. This indicates genetic variation for olfactory response to female odours. Parallel habitat marking therefore occurs at the intraspecific level in these two species, suggesting the possibility of an association with resource divergence.

Significant and repeatable habitat marking was found for two isofemale strains from the Melbourne population of D. melanogaster. This means that this trait is potentially amenable to genetic analysis.
\end{abstract}

\section{INTRODUCTION}

Closely related Drosophila species, e.g., the melanogaster subgroup, can be distinguished by ecologically important characteristics that can ultimately be related to the climate of the typical habitats of these species (Parsons, 1983a; Lachaise, 1983). Characteristics so far studied include resistance to heat, desiccation, and cold, the utilisation of resources in the wild, and the utilisation of the fermentation products ethanol and acetic acid under laboratory conditions. This approach can be taken to the intraspecific level (Parsons, 1983a) since these characteristics vary within and between populations of $D$. melanogaster as predicted from the climatic features of their habitats, especially for comparisons between populations from the temperate zone and humid tropics (Stanley and Parsons, 1981).

In addition to adaptation to climate and resources, flies may differ in their habitat preferences. For example, there are inter and intraspecific differences in olfactory attraction of flies to chemicals from feeding and breeding resources (West, 1961; Hoffmann and Parsons, 1984; Hoffmann, Parsons, and Nielsen, 1984). Furthermore, odours released by flies themselves may be important in resource attraction. Bartelt and Jackson (1984) described a male aggregation pheromone in $D$. virilis, which attracts both males

\footnotetext{
* Present address: Department of Genetics, University of
} California, Davis, CA 95616 , USA and females of any age. Spence, Hoffmann and Parsons (1984) demonstrated olfactory attraction of the sibling species Drosophila melanogaster and $D$. simulans to glass cylinders "marked" by female odours, with males preferring conspecific odours of virgin and non-virgin females. In this paper we extend these findings on habitat marking to the intraspecific level, using $D$. melanogaster and $D$. simulans from two widely separated and ecologically divergent Australian populations.

\section{MATERIALS AND METHODS}

Sympatric $D$. melanogaster and $D$. simulans populations were collected from an orchard near Melbourne, a temperate zone habitat at latitude $37^{\circ} \mathrm{S}$, and from suburban sites in Townsville, a tropical habitat at latitude $12^{\circ} \mathrm{S}$. Previously collected populations of these two species from Melbourne and Townsville have demonstrated considerable but parallel divergences for ecologically important characteristics especially ethanol tolerance (Parsons, 1983a for references). Populations were initiated by a minimum of 60 females from each location, and maintained on a sucrose dead yeast agar medium by mass transfer. The first trial was carried out with flies three generations removed from the field, and flies for subsequent trials came from generations immediately following. Five trials per species were carried out.

Following these experiments, five trials were carried out on two isofemale strains of $D$. 
melanogaster selected from the Melbourne population on the basis of being extremes for high and low ethanol tolerance out of 28 strains tested.

Flies were tested in a vertical wind tunnel olfactometer modified from a design by Wright (1966), according to the method described in Spence et al. (1984). The olfactometer tests mainly for optomotor anemotaxis, which is a mechanism whereby Drosophila respond to distant plant odours (Kellogg, Frizel and Wright, 1962; Shorey, 1976).

Glass cylinders were marked with odours from 600 non-virgin females (2-3 days old). We chose non-virgin females, since virgin females and males, while still acting as attractants, were less effective (Spence et al., 1984). Females were discarded after

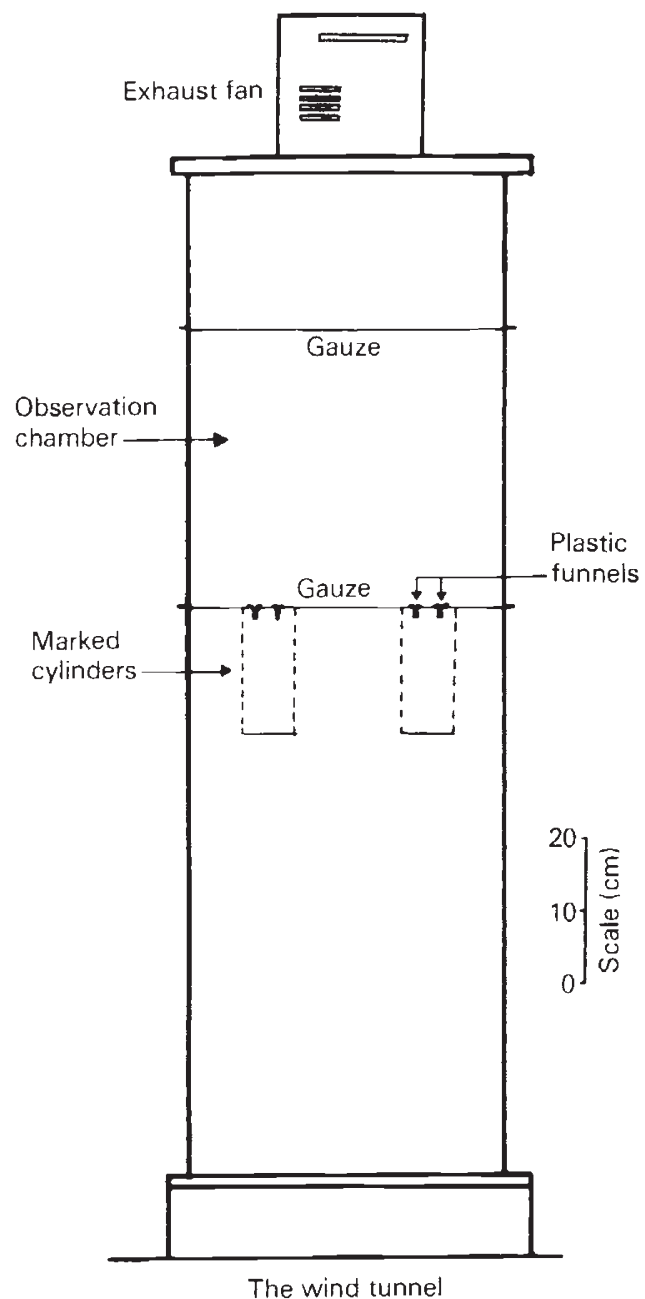

Figure 1 Simplified diagram to show important features of the wind tunnel mentioned in the text. More precise specifications appear in Spence et al, (1984).
8 hours, and the "marked" cylinders covered with gauze at both ends were then placed into the wind tunnel (fig. 1). Three hundred males and females from each population (males only in the isofemale strain experiments) were then simultaneously released into the observation chamber of the tunnel; each population was marked with a different fluorescent dust, and the colours were alternated between experiments. Odours were drawn from the marked cylinders into the observation chamber by means of an exhaust fan, the entry of flies being permitted through two plastic funnels. The cylinders served as traps, and flies were accumulated overnight.

\section{RESULTS}

Data for the two species are presented in tables 1 and 2. Both sexes are attracted to the residual odours in the marked cylinders, since fewer than 10 flies are normally retrieved from unmarked cylinders. Fewer females than males were captured in the marked cylinders, which is consistent with the interspecific results of Spence et al., (1984). Chi-square contingency analyses were carried out separately for each trial, to test for independence among the residual odours and captured flies of the two populations. For $D$. melanogaster males, the data indicate a significant association for all trials. A heterogeneity $\chi^{2}$ among trials was computed by taking the $\chi^{2}$ value for the pooled results from the summed value across trials, and was not significant $\left(\chi^{2}=2 \cdot 67\right.$, df $\left.4, p<0 \cdot 05\right)$. For D. simulans males, 3 out of 5 trials and the pooled results show significance, and there is no heterogeneity among trials $\left(\chi^{2}=3 \cdot 77\right.$, df $4, p>$ $0.05)$. In contrast, data for $D$. melanogaster and D. simulans females show no significant departures from homogeneity in any trial.

The degree of association among odours and populations can be described by the following index of choice difference:

$$
\Delta=P_{m \mid m}-P_{m \mid t}
$$

where $P_{m \mid m}=P$ (capture in Melbourne trap $\mid$ Melbourne population) and $P_{\left.m\right|_{t}}=P$ (capture in Melbourne trap/Townsville population). This index was used by Turelli, Coyne and Prout (1984) to measure the association among flies captured and recaptured on different fruit types, and has a range from -1 to +1 , with values greater than zero indicating a positive association. The probability estimate $P_{m \mid m}$ is the number of Melbourne flies captured in the Melbourne cylinder, divided by 
Table 1 Attraction of $D$. melanogaster to residual odours from Melbourne and Townsville non-virgin females (numbers in brackets are expected, under a $2 \times 2$ test of homogeneity)

\begin{tabular}{|c|c|c|c|c|c|c|c|c|c|}
\hline & \multirow{3}{*}{$\begin{array}{l}\text { Population } \\
\text { Odour } \\
\text { (Attractant) }\end{array}$} & \multirow{2}{*}{\multicolumn{5}{|c|}{ Flies Captured }} & \multirow{2}{*}{\multicolumn{2}{|c|}{ Females }} & \multirow[b]{3}{*}{$\Delta \pm S E^{\mathrm{a}}$} \\
\hline & & & & & & & & & \\
\hline & & Melbourne & Townsville & $\chi_{1}^{2}$ & $\Delta \pm \mathrm{SE}^{\mathrm{a}}$ & Melbourne & Townsville & $\chi_{1}^{2}$ & \\
\hline \multirow{2}{*}{ Trial 1} & Melbourne & $35(29 \cdot 1)$ & $30(35.9)$ & & & $6(5 \cdot 7)$ & $6(6 \cdot 3)$ & & \\
\hline & Townsville & $25(30.9)$ & $44(38 \cdot 1)$ & $4 \cdot 20^{*}$ & $0.18 \pm 0.08$ & $16(16 \cdot 3)$ & $18(17 \cdot 7)$ & 0.03 & $0.02 \pm 0.13$ \\
\hline \multirow[t]{2}{*}{ Trial 2} & Melbourne & $28(22 \cdot 3)$ & $20(25 \cdot 7)$ & & & $14(13 \cdot 4)$ & $12(12 \cdot 6)$ & & \\
\hline & Townsville & $18(23 \cdot 7)$ & $33(27 \cdot 3)$ & $5 \cdot 28^{*}$ & $0.23 \pm 0.10$ & $5(5 \cdot 6)$ & $6(5 \cdot 4)$ & $0 \cdot 22$ & $0 \cdot 07 \pm 0 \cdot 15$ \\
\hline \multirow[t]{2}{*}{ Trial 3} & Melbourne & $40(30 \cdot 6)$ & $20(29 \cdot 4)$ & & & $8(9 \cdot 2)$ & $18(16 \cdot 8)$ & & \\
\hline & Townsville & $12(21 \cdot 4)$ & $30(20 \cdot 6)$ & $14 \cdot 35^{* *}$ & $0.40 \pm 0.09$ & $4(2 \cdot 8)$ & $4(5 \cdot 2)$ & $-{ }^{b}$ & $-0 \cdot 15 \pm 0 \cdot 16$ \\
\hline \multirow[t]{2}{*}{ Trial 4} & Melbourne & $16(11 \cdot 3)$ & $22(26 \cdot 7)$ & & & $3(2 \cdot 0)$ & $14(15 \cdot 0)$ & & \\
\hline & Townsville & $8(12 \cdot 7)$ & $35(30 \cdot 3)$ & $5 \cdot 34^{*}$ & $0.28 \pm 0.11$ & $1(2 \cdot 0)$ & $16(15 \cdot 0)$ & $-^{b}$ & $0 \cdot 28^{c}$ \\
\hline \multirow[t]{2}{*}{ Trial 5} & Melbourne & $13(9 \cdot 8)$ & $4(7 \cdot 2)$ & & & $2(1.7)$ & $4(4 \cdot 3)$ & & \\
\hline & Townsville & $10(13 \cdot 2)$ & $13(9 \cdot 8)$ & $4 \cdot 35^{*}$ & $0 \cdot 33 \pm 0 \cdot 15$ & $2(2 \cdot 3)$ & $6(5 \cdot 7)$ & $-^{\mathrm{b}}$ & $0 \cdot 10^{c}$ \\
\hline \multirow[t]{2}{*}{ Total } & Melbourne & $132(102 \cdot 5)$ & $96(125 \cdot 5)$ & & & $33(32 \cdot 2)$ & $54(54.8)$ & & \\
\hline & Townsville & $73(102 \cdot 5)$ & $155(125 \cdot 5)$ & $30 \cdot 85^{* *}$ & $0.26 \pm 0.04$ & $28(28.8)$ & $50(49 \cdot 2)$ & 0.07 & $0.02 \pm 0.08$ \\
\hline
\end{tabular}

${ }^{a} \Delta=P$ (Capture in Melbourne trap/Melbourne population) - $P$ (Capture in Melbourne trap/Townsville population).

$* P<0.05$.

** $P<0.01$.

${ }^{b}$ Fishers exact test, $P<0 \cdot 05$.

${ }^{c}$ Standard error not given since total captured for any population is less than 5 .

Table 2 Attraction of $D$. simulans to residual odours from Melbourne and Townsville non-virgin females (number in brackets are expected, under a $2 \times 2$ test of homogeneity)

\begin{tabular}{|c|c|c|c|c|c|c|c|c|c|}
\hline & \multirow{3}{*}{$\begin{array}{l}\text { Population } \\
\text { odour } \\
\text { (Attractant) }\end{array}$} & \multirow{2}{*}{\multicolumn{5}{|c|}{ Flies Captured }} & \multirow{2}{*}{\multicolumn{3}{|c|}{ Females }} \\
\hline & & & & & & & & & \\
\hline & & Melbourne & Townsville & $\chi_{1}^{2}$ & $\Delta \pm \mathrm{SE}^{\mathrm{a}}$ & Melbourne & Townsville & $\chi_{1}^{2}$ & $\Delta \pm \mathrm{SE}^{\mathrm{a}}$ \\
\hline \multirow[t]{2}{*}{ Trial 1} & Melbourne & $32(31 \cdot 0)$ & $12(13 \cdot 0)$ & & & $26(26 \cdot 7)$ & $8(7 \cdot 3)$ & & \\
\hline & Townsville & $18(19 \cdot 0)$ & $9(8 \cdot 0)$ & $0 \cdot 29$ & $0.07 \pm 0.13$ & $14(13 \cdot 3)$ & $3(3 \cdot 7)$ & $-^{b}$ & $-0.08 \pm 0.15$ \\
\hline \multirow[t]{2}{*}{ Trial 2} & Melbourne & $26(22 \cdot 2)$ & $7(10 \cdot 8)$ & & & $10(9 \cdot 4)$ & $6(6 \cdot 6)$ & & \\
\hline & Townsville & $19(22 \cdot 8)$ & $15(11 \cdot 2)$ & $3 \cdot 98^{*}$ & $0 \cdot 26 \pm 0 \cdot 12$ & $7(7 \cdot 6)$ & $6(5 \cdot 4)$ & $0 \cdot 22$ & $0.09 \pm 0.19$ \\
\hline \multirow[t]{2}{*}{ Trial 3} & Melbourne & $15(10 \cdot 2)$ & $7(11 \cdot 8)$ & & & $8(5 \cdot 9)$ & $6(8 \cdot 1)$ & & \\
\hline & Townsville & $10(14 \cdot 8)$ & $22(17 \cdot 2)$ & $7 \cdot 15^{* *}$ & $0.36 \pm 0.13$ & $3(7 \cdot 1)$ & $12(9.9)$ & $2 \cdot 42$ & $0.39 \pm 0.17$ \\
\hline \multirow[t]{2}{*}{ Trial 4} & Melbourne & $26(22 \cdot 6)$ & $12(15.4)$ & & & $15(14 \cdot 7)$ & $10(10.3)$ & & \\
\hline & Townsville & $15(18 \cdot 4)$ & $16(12 \cdot 6)$ & $2 \cdot 84$ & $0 \cdot 21 \pm 0 \cdot 12$ & $8(8 \cdot 3)$ & $6(5 \cdot 7)$ & 0.03 & $0.03 \pm 0.16$ \\
\hline \multirow[t]{2}{*}{ Trial 5} & Melbourne & $55(50 \cdot 2)$ & $8(12 \cdot 8)$ & & & $28(30 \cdot 2)$ & $17(14 \cdot 8)$ & & \\
\hline & Townsville & $35(39 \cdot 8)$ & $15(10 \cdot 2)$ & $5 \cdot 15^{*}$ & $0.26 \pm 0 \cdot 11$ & $15(12 \cdot 8)$ & $4(6 \cdot 2)$ & 1.69 & $-0.16 \pm 0.11$ \\
\hline \multirow[t]{2}{*}{ Total } & Melbourne & $154(135.9)$ & $46(64 \cdot 1)$ & & & $87(83.9)$ & $42(45 \cdot 1)$ & & \\
\hline & Townsville & $107(125 \cdot 1)$ & $77(58.9)$ & $15 \cdot 64^{* *}$ & $0.22 \pm 0.05$ & $49(52 \cdot 1)$ & $31(27.9)$ & 0.83 & $0.06 \pm 0.07$ \\
\hline
\end{tabular}

See table I for footnotes.

the total number of Melbourne flies captured and similarly $P_{m \mid t}$ is the number of Townsville flies captured in the Melbourne cylinder, divided by the total number of Townsville flies captured. The standard errors of $\Delta$ are approximated by the square root of the sum of the sample variances for each conditional probability, provided that the number captured from each population exceeds five (Turelli et al., 1984). For the male data, estimates are positive for all trials in both species, and significantly greater than zero for the pooled results, while estimates from female data tend to vary in sign and do not differ significantly from zero (tables 1,2 ), which are results consistent with the $\chi^{2}$ analyses.

Data for the two Melbourne isofemale strains are presented in table 3 . Because of the insignificant female effects in tables 1 and 2, they were left out of these experiments leaving the attraction of males to the residual odours of females. Two of the five trials and the pooled results show significance and in all cases $\Delta>0$. The heterogeneity $\chi_{4}^{2}$ among trials came to 3.91 which is not significant. 
Table 3 Attraction of $D$. melanogaster males to residual odours from non-virgin females of two isofemale strains from the Melbourne population (numbers in brackets are expected, under a $2 \times 2$ test of homogeneity)

\begin{tabular}{|c|c|c|c|c|c|}
\hline Trial & $\begin{array}{l}\text { Population Odour } \\
\text { (Attractant) }\end{array}$ & $\begin{array}{l}\text { Isofemale strain } \\
1\end{array}$ & 2 & $x_{1}^{2}$ & $\Delta+\mathrm{SE}^{\mathrm{a}}$ \\
\hline \multirow[t]{2}{*}{1} & 1 & $34(32 \cdot 6)$ & $12(13 \cdot 4)$ & \multirow[t]{2}{*}{$0 \cdot 34$} & \multirow[t]{2}{*}{$0.07 \pm 0.11$} \\
\hline & 2 & $32(33 \cdot 4)$ & $15(13 \cdot 6)$ & & \\
\hline \multirow[t]{2}{*}{2} & 1 & $58(53.9)$ & $19(23 \cdot 1)$ & \multirow[t]{2}{*}{$2 \cdot 31$} & \multirow[t]{2}{*}{$0.14 \pm 0.09$} \\
\hline & 2 & $40(44-1)$ & $23(18 \cdot 9)$ & & \\
\hline \multirow[t]{2}{*}{3} & 1 & $18(14 \cdot 0)$ & $7(11 \cdot 0)$ & \multirow[t]{2}{*}{$5 \cdot 19^{*}$} & \multirow[t]{2}{*}{$0 \cdot 32 \pm 0.13$} \\
\hline & 2 & $10(14 \cdot 0)$ & $15(11 \cdot 0)$ & & \\
\hline \multirow[t]{2}{*}{4} & 1 & $26(21 \cdot 9)$ & $16(20 \cdot 1)$ & \multirow[t]{2}{*}{$2 \cdot 87$} & \multirow{2}{*}{$0 \cdot 17 \pm 0 \cdot 10$} \\
\hline & 2 & $25(29 \cdot 1)$ & $31(26 \cdot 9)$ & & \\
\hline \multirow[t]{2}{*}{5} & 1 & $39(32 \cdot 7)$ & $33(39 \cdot 3)$ & \multirow[t]{2}{*}{$5 \cdot 68 *$} & \multirow[t]{2}{*}{$0.21 \pm 0.09$} \\
\hline & 2 & $15(21 \cdot 3)$ & $32(25 \cdot 7)$ & & \\
\hline \multirow[t]{2}{*}{ Total } & 1 & $175(155 \cdot 6)$ & $87(106 \cdot 4)$ & \multirow[t]{2}{*}{$12 \cdot 48^{* *}$} & \multirow[t]{2}{*}{$0.16 \pm 0.04$} \\
\hline & 2 & $122(141 \cdot 4)$ & $116(96.6)$ & & \\
\hline
\end{tabular}

See table 1 for footnotes.

\section{DISCUSSION}

Males are preferentially attracted to residual odours from females of their own populations in both of these cosmopolitan species (tables 1 and 2). This phenomenon may arise in a number of ways. Males may be differentially attracted to female odours which would imply differences in both odour recognition and production by females. Alternatively, males may prefer odours of all flies. However, the experiments described in Spence et al., (1984) indicate that flies are only slightly attracted to residual odours of males. Finally, males may be attracted to substances they transmit during copulation. This seems unlikely, since Spence et al., (1984) found that D. melanogaster and $D$. simulans males were differentially attracted to residual odours of virgin and non-virgin females. Hence the data suggest variation for olfactory detection by males, rather than variation in odour production by this sex.

These interpopulation differences have a genetic basis, since they were detected after culturing flies for several generations under the same conditions, and there was no heterogeneity across generations. The estimates of association obtained in the present study can be compared with the interspecific results obtained by Spence et al., (1984) in the same apparatus. Estimates for $\Delta$ are $0.50 \pm 0 \cdot 10,0.45 \pm 0.12$ and $0.52 \pm 0.13$ for three comparable trials, which are about twice the values given in tables 1 and 2, even though the number of flies caught in the trap cylinders were similar in both experiments. Hence the preference for female odours from Melbourne and Townsville populations is less than observed for $D$. melanogaster and $D$. simulans. This is qualitatively consistent with the extent to which these populations have diverged genetically relative to the sibling species (Parsons, 1983b).

Chemical stimuli from mature Drosophila females are important in initiating courtship of males (Tomkins, Hall and Hall, 1980; Venard and Jallon, 1980; Antony and Jallon 1982). These stimuli consist of apparently species-specific longchain hydrocarbons in the cuticle of females, and there are recent suggestions of some genetic variation within species (Jallon, 1984; Tomkins and Hall, 1984). The previously reported phenomenon of interspecific habitat marking in $D$. melanogaster and $D$. simulans could be related to these stimuli (Spence et al., 1984). However, many of these chemicals have low volatility and may only affect male behaviours over a few millimeters, probably being detected by contact rather than olfaction (Antony and Jallon, 1982).

On the other hand, following suggestions concerning preferences by rare Drosophila males (Ehrman and Probber, 1978), habitat marking could alternatively or additionally be the consequence of a recognition system based on compounds that are normal metabolic products (see Parsons, $1983 b$ ). In this way, females from different populations would leave a characteristic bouquet preferentially attracting males to the bouquet of a given population. These recognition compounds may be normal fermentation products, attractive over relatively long distances, and in this light the parallel results for the sibling species are expected. On the basis of this interpretation, it follows that isofemale strains within a population that are extreme for metabolic phenotypes may be 
expected to show this type of marking. This may explain the isofemale data (table 3 ), although we have not demonstrated an association between habitat marking and ethanol metabolism per se. The $\Delta=0.16$ for the isofemale strain marking experiments is lower than $\Delta=0.26$ obtained for $D$. melanogaster at the interpopulation level as expected on the basis of metabolic divergence. Irrespective of the validity of the interpretation involving metabolic phenotypes, the important point is the demonstration of habitat marking within a population, which means that this trait is amenable to more sophisticated genetic analyses based upon isofemale strains (Parsons, 1980).

The preferential attraction of males may contribute to some sexual isolation, especially as the courtship behaviours of cosmopolitan Drosophila species tend to be associated with resources (Spieth, 1952). Tests of sexual isolation among Drosophila populations are usually carried out in mating chambers and these often indicate that isolation is weak or absent especially in $D$. melanogaster (Spieth and Ringo, 1983). However, the behaviours described here are unlikely to be important in mating chamber tests, and it seems worthwhile to further investigate isolation with paradigms incorporating habitat marking. In any case it can be hypothesized that if habitat marking occurs in the wild, this may increase sexual isolation at the microhabitat level. In addition there may be increased competition among males for mates enhancing levels of sexual selection among males perhaps promoting the high levels of multiple insemination usual in populations of $D$. melanogaster and other widespread Drosophila species (Anderson, 1974; Milkman and Zietler, 1974).

Acknowledgments We are grateful to Janine Clarke and Garry Spence for technical assistance.

\section{REFERENCES}

ANDERSON, w. W. 1974. Frequent multiple insemination in a natural population of Drosophila pseudoobscura. Am. Nat., $108,709-711$.

ANTONY, C. AND JALLON, J. M. 1982. The chemical basis for sex recognition in Drosophila melanogaster. J. Insect Physiol., 28, 873-880.
BARTELT, R. J. AND JACKSON, L. L. 1984. Hydrocarbon component of the Drosophila virilis (Diptera: Drosophilidae) aggregation pheromone: (Z)-10-heneicosene. Ann. Ent. Soc. Am., 77, 367-371.

EHRMAN, L. AND PRobBer, J. 1978. Rare Drosophila males; the mysterious matter of choice. Am. Scientist, 66, 216-222.

HOFFMANN, A. A. AND PARSONS, P. A. 1984. Olfactory response and resource utilization in Drosophila: interspecific comparisons. Biol. J. Linn. Soc., 22, 43-53.

HOFFMANN, A. A., PARSONS, P. A. AND NIELSON, K. M. 1984. Habitat selection: olfactory response of Drosophila melanogaster depends on resources. Heredity, 53, 139-145.

JALLON, J. M. 1984. A few chemical words exchanged by drosophiles during courtship. Behav. Genet. (in press).

KELLOGG, F. E., FRIZEL, D. E. AND WRIGHT, R. H. 1962. The olfactory guidance of insects. IV. Drosophila. Canadian Entomol., 94, 884-888.

LACHAISE, D. 1983. Reproductive allocation in tropical Drosophilidae: further evidence on the role of breeding-site choice. Am. Nat., 122, 132-146.

MILKMAN, R. D. AND ZEITLER, R. D. 1974. Concurrent multiple paternity in natural and laboratory populations of Drosophila melanogaster. Genetics, 78, 1191-1193.

PARSONS, P. A. 1980. Isofemale strains and evolutionary strategies in natural populations. Evol. Biol., 13, 175-217.

PARSONS, P. A. 1983a. The Evolutionary Biology of Colonizing Species. Cambridge University Press, New York.

PARSONS, P. A. 1983 b. Ecobehavioral genetics: habitats and colonists. Ann. Rev. Ecol. Syst., 14, 35-55.

Shorey, H. H. 1976. Animal Communication by Pheromones. Academic Press, New York.

SPIETH, H. T. 1952. Mating behavior within the genus Drosophila (Diptera). Bull. Am. Mus. Nat. Hist., 99, 401-474.

SPIETH, H. T. AND RINGO, J. M. 1983. Mating behavior and sexual isolation in Drosophila. In The Genetics and Biology of Drosophila, Vol. 3c. (Eds.) M. Ashburner, Carson, H. L. and Thompson, J. N. Academic Press, London.

SPENCE, G. E., hOFFMANN, A. A. AND PARSONS, P. A. 1984. Habitat marking: males attracted to residual odors of two Drosophila species. Experientia, 40, 763-765.

STANLEY, S. M. AND PARSONS, P. A. 1981. The response of the cosmopolitan species Drosophila melanogaster to ecological gradients. Proc. Ecol. Soc. Aust., 11, 121-130.

TOMK1NS, L., HALL, J. C. AND HALL, L. M. 1980. Courtship stimulating volatile compounds from normal and mutant Drosophila. J. Insect Physiol., 26, 689-697.

TOMKINS, L. AND HALL, J. C. 1984. Sex pheromones enable Drosophila males to discriminate between conspecific females from different laboratory stocks. Anim. Behav., 32, 349-352.

TURElli, M., COYNE, J. A. AND PROUT, T. 1984. Resource choice in orchard populations of Drosophila. Biol. J. Linn. Soc., 22, 95-106.

VENARD, R. AND JALLON, J. M. 1980. Evidence for an aphrodisiac pheromone of female Drosophila. Experientia, $36,211-212$.

WEST, A. S. 1961. Chemical attractants for adult Drosophila species. J. Econ. Entomol., 54, 677-681.

WRIGHT, R. H. 1966. An insect olfactometer. Canadian Entomol., 98, 282-285. 